\title{
The Inflammatory Bowel Disease (IBD) BioResource: an open-access platform of over 25,000 patients to accelerate research in Crohn's and Colitis
}

Successes in IBD genetics provide an opportunity to tease out aetiopathogenesis, develop new treatments and test for stratified medicine. In particular, identifying the effects of genetics variations on nutrient-gene interactions (Nutrigenetics) and looking at the impact of nutrition globally on nutrient-gene interactions (Nutrigenomics) may contribute to developing personalised nutrition for disease prevention and treatment. However, ALL require access to large cohorts of genetically and clinically well-characterised patients. To that effect, the UK IBD Genetics Consortium and the NIHR BioResource launched IBD BioResource ${ }^{(1)}$ in 2016. The aim at outset was to build a large national platform of individuals with Crohn's disease (CD) or ulcerative colitis (UC) and to generate detailed genetic and phenotypic data to facilitate downstream translational research.

The Main cohort comprises individuals with established CD and UC. Both clinical and self-reported phenotype data are collected, alongside plasma, serum and DNA samples. The Inception cohort recruits newly diagnosed IBD patients and provide detailed sampling unconfounded by drug treatment or effects of surgery. The IBD BioResource panel is accessible to any investigators from science to industry for data / samples or recall of genotype-selected participants to donate further samples or trial novel therapies.

The main cohort has now over 27,000 individuals enrolled into the IBD BioResource with rich phenotypic data and DNA samples undergoing Whole Genome/Exome Sequencing as well as Genome Wide Association Scanning. Inception cohort: 60 hospital sites have been trained to identify and recruit to this cohort. Recruitment is going well too with $\sim 25 \%$ of our ' 1,000 ' target reached. One measure of success for IBD BioResource is the use of its panel for the facilitation of IBD research. To date 12 'Stage 2 studies' have applied to utilise the IBD BioResource: $\sim 40 \%$ requested access to anonymised samples and data while the remaining $\sim 60 \%$ required involvement of participants. Focus of studies ranged from disease mechanisms and immunology through to genetic, environmental and microbial interactions.

The IBD BioResource and its network are on course to facilitating IBD Research through access to their panel. We are yet to accommodate studies with a nutritional focus and therefore strongly welcome formal applications.

1. Parkes M (2019) IBD BioResource Investigators. Gut Sep; 68(9), 1537-1540. 